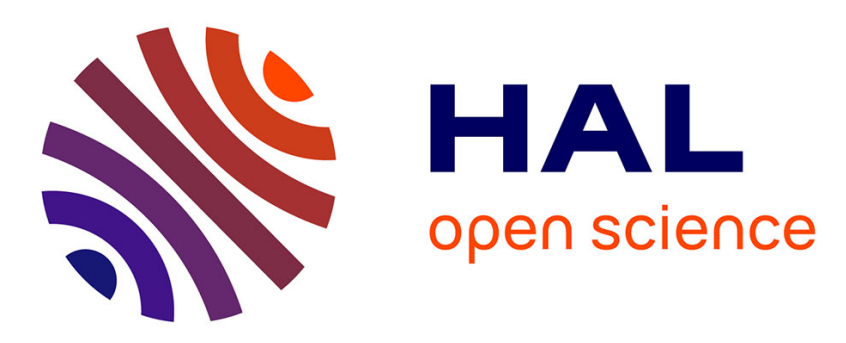

\title{
Non parametric modelling of ECG: Applications to denoising and single sensor fetal ECG extraction
}

Bertrand Rivet, Mohammad Niknazar, Christian Jutten

\section{To cite this version:}

Bertrand Rivet, Mohammad Niknazar, Christian Jutten. Non parametric modelling of ECG: Applications to denoising and single sensor fetal ECG extraction. LVA/ICA 2012 - 10th International Conference on Latent Variable Analysis and Signal Separation, Mar 2012, Tel-Aviv, Israel. pp.470-477. hal-00681534

\section{HAL Id: hal-00681534 https://hal.science/hal-00681534}

Submitted on 21 Mar 2012

HAL is a multi-disciplinary open access archive for the deposit and dissemination of scientific research documents, whether they are published or not. The documents may come from teaching and research institutions in France or abroad, or from public or private research centers.
L'archive ouverte pluridisciplinaire HAL, est destinée au dépôt et à la diffusion de documents scientifiques de niveau recherche, publiés ou non, émanant des établissements d'enseignement et de recherche français ou étrangers, des laboratoires publics ou privés. 


\title{
Nonparametric modelling of ECG: Applications to denoising and to single sensor fetal ECG extraction
}

\author{
Bertrand Rivet, Mohammad Niknazar, and Christian Jutten ${ }^{\star}$ \\ GIPSA-Lab , CNRS UMR-5216, University of Grenoble \\ Domaine Universitaire, BP 46, 38402 Saint Martin d'Hères cedex, France
}

\begin{abstract}
In this work, we tackle the problem of fetal electrocardiogram (ECG) extraction from a single sensor. The proposed method is based on non-parametric modelling of the ECG signal described thanks to its second order statistics. Each assumed source in the mixture is thus modelled as a second order process thanks to its covariance function. This modelling allows to reconstruct each source by maximizing the related posterior distribution. The proposed method is tested on synthetic data to evaluate its performance behavior to denoise ECG. It is then applied on real data to extract fetal ECG from a single maternal abdominal sensor.
\end{abstract}

Key words: non-parametric modelling, source extraction, denoising, fetal ECG extraction.

\section{Introduction}

Fetal electrocardiogram (f-ECG) extraction from maternal abdominal ECG sensors is an old problem. Since the first works of Cremer [1] who produced a very primitive record of fetal rate activity, this problem is still of interest nowadays since it is a fascinating issue due to the characteristics of the involved signals. Indeed, the f-ECG is definitively less powerful than the mother's ECG (m-ECG), moreover the recorded signals are also contaminated by noise due to electromyogram (EMG) or to power line interference and they are also influenced by the fluctuation of the baseline. Among the several approaches used to tackle the extraction of f-ECG, one can quote methods which require several sensors e.g., adaptive filtering [15], blind source separation $[2,16]$ or quasi-periodic analysis [14].

In this paper, we consider the same issue but assuming that only a single sensor is available. In this case, one can extract f-ECG by singular value decomposition [4] or by nonlinear decomposition such as shrinkage wavelet denoising [7] or nonlinear projections [10]. Moreover, state modelling as Kalman filtering [13] has been applied to overcome the lack of information provided by a single sensor.

\footnotetext{
^ Christian Jutten is also with Institut Universitaire de France.
} 


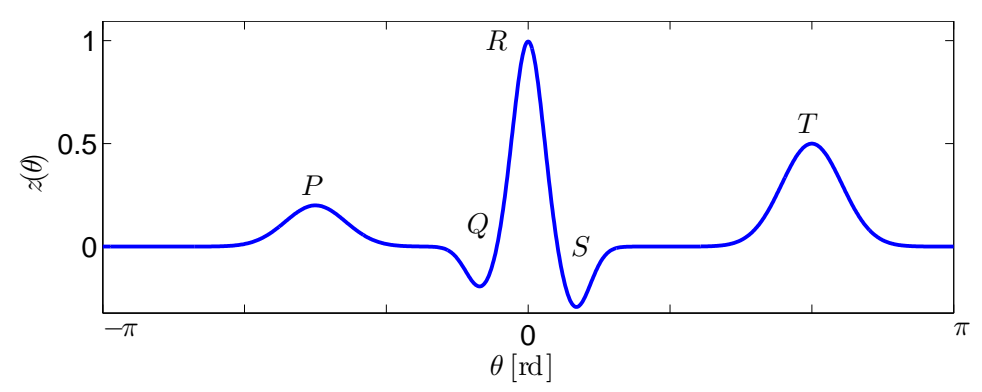

Fig. 1. Modelling (1) of the amplitude of one beat.

Among these methods, the latter has been shown to be the most efficient [12]. However, Kalman filtering relies on a very strong assumption: the state equation, which models the dynamical evolution of the unobserved state. As a consequence, Kalman filtering needs reliable prior about the state to perform accurately. To overcome the potential lack of prior information about the system, we propose in this study to model the second order statistics of the signal instead of the signal itself.

This article is organized as follows. Section 2 presents the proposed approach to model a signal thanks to its second order statistics. The proposed algorithm to extract f-ECG is then introduced in Section 3. Numerical experiments and results are given in Section 4 before conclusion and perspectives in Section 5 .

\section{Nonparametric modelling of ECG}

As already proposed in [13], one can choose a parametric model of ECG: each beat of an ECG signal is a summation of 5 Gaussian functions, each of them modelling the P, Q, R, S and T waves as illustrated in Figure 1:

$$
z(\theta)=\sum_{i \in\{P, Q, R, S, T\}} a_{i} \exp \left(-\frac{\left(\theta-\theta_{i}\right)^{2}}{2 \sigma_{i}^{2}}\right),
$$

where $a_{i}, \theta_{i}$ and $\sigma_{i}$ are the amplitude, the position and the width of each wave, respectively. Note that in this model, the beats are defined in phase $\theta \in[-\pi, \pi]$, so that each beat is assumed to have a linear variation of phase with respect to the time, even if each beat has not the same duration. This model can then be used in an extended Kalman filtering to denoise a single ECG or extract f-ECG from a mixture of $\mathrm{m}-\mathrm{ECG}$ and $\mathrm{f}-\mathrm{ECG}[13,11]$. This method is thus a parametric method since the unknown amplitude $z(\theta)$ is explicitly parameterized.

On the other hand, nonparametric methods perform estimation, prediction or denoising without explicitly parameterizing the unknown amplitude $z(\theta)$. For instance, a well known approach is the spline smoothing [5]. If one considers the ECG $z(\theta)$ as a statistical process, it can be fully described at the second order by 

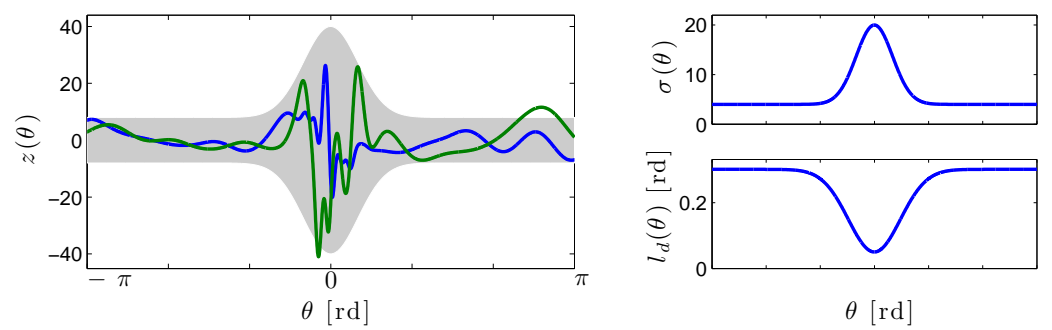

Fig. 2. Two functions drawn at random from a zero-mean GP with covariance function (2). The shaded area represents plus and minus two times the standard deviation for the prior. On the right, the related $\sigma(\theta)$ and $l_{d}(\theta)$ functions.

its mean function $m(\theta)=\mathbb{E}[z(\theta)]$ and covariance function $k\left(\theta_{1}, \theta_{2}\right) \triangleq \mathbb{E}\left[\left(z\left(\theta_{1}\right)-\right.\right.$ $\left.\left.m\left(\theta_{1}\right)\right)\left(z\left(\theta_{2}\right)-m\left(\theta_{2}\right)\right)\right][8]$. Obviously, the ECG signal is almost surely a more complex statistical process than a simple second order one. As a consequence, considering only its second order statistics, it relies among the Gaussian process (GP) framework which is widely used in machine learning e.g., [6,9]. A GP $z(\theta)$ is a distribution over functions denoted as $\mathcal{G P}\left(m(\theta), k\left(\theta_{1}, \theta_{2}\right)\right)$. In this case, the statistical latent process $z(\theta)$ is not directly parameterized as in parametric model, but its statistics are it thanks to hyper-parameters. This means that one has to choose a class of semidefinite positive functions $k\left(\theta_{1}, \theta_{2}\right)$ which describes the expected second order properties of the latent process.

In this study, we propose to use the following non-stationary covariance function

$$
k\left(\theta_{1}, \theta_{2}\right)=\sigma\left(\theta_{1}\right) \sigma\left(\theta_{2}\right) \sqrt{\frac{2 l_{d}\left(\theta_{1}\right) l_{d}\left(\theta_{2}\right)}{l_{d}\left(\theta_{1}\right)^{2}+l_{d}\left(\theta_{2}\right)^{2}}} \exp \left(-\frac{\left(\theta_{1}-\theta_{2}\right)^{2}}{l_{d}\left(\theta_{1}\right)^{2}+l_{d}\left(\theta_{2}\right)^{2}}\right),
$$

with

$$
\begin{aligned}
& \sigma(\theta)=a_{m}+\left(a_{M}-a_{m}\right) \exp \left(-\frac{\left(\theta-\theta_{0}\right)^{2}}{2 \sigma_{T}^{2}}\right), \\
& l_{d}(\theta)=l_{M}-\left(l_{M}-l_{m}\right) \exp \left(-\frac{\left(\theta-\theta_{0}\right)^{2}}{2 \sigma_{l}^{2}}\right),
\end{aligned}
$$

where $\sigma(\theta)$ and $l_{d}(\theta)$ allow to have a time-varying power (between $a_{m}$ and $a_{M}$ ) and a time-varying length scale correlation (between $l_{m}$ and $l_{M}$ ), respectively. Indeed, as shown in Fig. 1, an ECG beat can be decomposed into three parts: the $\mathrm{P}$ wave, the QRS complex and the $\mathrm{T}$ wave. The $\mathrm{P}$ and $\mathrm{T}$ waves share the same kind of second order statistics: a larger length scale and a lower power than the QRS complex. Fig. 2 shows two functions drawn at random from the zero-mean GP prior with covariance function (2). This figure illustrates the flexibility of such a representation compared to model (1) since with the same prior 
$\mathcal{G P}\left(0, k\left(\theta_{1}, \theta_{2}\right)\right)$, it can generate a multitude of different shapes with the same prior.

\section{Denoising of ECG and extraction of fetal ECG from a single sensor}

Suppose that the observed values $x(t)$ differ from the ECG, $s(t)$, by additive noise $n(t)$ :

$$
x(t)=s(t)+n(t),
$$

and that this noise is uncorrelated with $s(t)$. The aim of this study is to infer the values of $s(t)$ from $x(t)$, i.e. to denoise or extract the ECG from the observations. Moreover, it is assumed that the ECG signal, $s(t)$, is a succession of beats, each of them following a zero-mean GP defining by (2) and that the additive noise follows a zero-mean GP whose covariance function $k_{n}\left(t, t^{\prime}\right)$ is given by

$$
k_{n}\left(t, t^{\prime}\right)=\sigma_{n}^{2} \exp \left(-\frac{\left(t-t^{\prime}\right)^{2}}{2 l_{n}^{2}}\right)+\sigma_{w}^{2} \delta\left(t-t^{\prime}\right),
$$

where $\delta(\cdot)$ is the delta Dirac function. In this expression, the first term is useful for instance to model a baseline variation as a stationary process for which the correlation is almost unity between close samples and decreases as their distance increases compared to the length scale $l_{n}$. The second term models a white Gaussian noise of power $\sigma_{w}^{2}$. From (3) and (4), the covariance function of observation $x(t)$ is thus expressed as

$$
k_{x}\left(t, t^{\prime}\right)=k_{s}\left(t, t^{\prime}\right)+k_{n}\left(t, t^{\prime}\right),
$$

where

$$
k_{s}\left(t, t^{\prime}\right)=\sum_{n=1}^{N} \sum_{n^{\prime}=1}^{N} k\left(t-\tau_{n}, t^{\prime}-\tau_{n^{\prime}}\right)
$$

and $\left\{\tau_{n}\right\}_{1 \leq n \leq N}$ is the set of $\mathrm{R}$ peak instants that can be estimated easily from the raw signals. From this modelling and assuming that the observed process $x(t)$ has been recorded at times $\left\{T_{m}\right\}_{1 \leq m \leq M}$, the covariance matrix of this process is thus given by $K_{x}$, whose $(i, j) t h$ entry is

$$
K_{x}(i, j)=k_{x}\left(T_{i}, T_{j}\right) .
$$

One can then infer on the value $s(t)$ thanks to the maximization of the a posteriori distribution of $s(t)$ given $\mathbf{x}=\left[x\left(T_{1}\right), \cdots, x\left(T_{M}\right)\right]^{T}$ by

$$
\hat{s}(t)=\mathbf{k}^{T} K_{x}^{-1} \mathbf{x},
$$

where $\mathbf{k}=\left[k\left(t, T_{1}\right), \cdots, k\left(t, T_{M}\right)\right]^{T}$. It is interesting to note that as soon as $\sigma_{w}^{2} \neq 0$, matrix $K_{x}$ is invertible as the summation of definite positive matrices and a diagonal matrix $\sigma_{w}^{2} I$. This algorithm needs some comments. First of all, 
the recorded signal, $x(t)$, does not need to be regularly sampled and one can observe from (7) that the value of the latent process, $s(t)$, can be predicted at any time $t$ even for $t \neq T_{i}, \forall i \in\left\{1, \cdots, T_{M}\right\}$. Moreover, the hyper-parameters $\boldsymbol{\theta}=$ $\left\{a_{m}, a_{M}, \sigma_{T}^{2}, l_{m}, l_{M}, \sigma_{l}^{2}, \theta_{0},\left\{\tau_{k}\right\}_{k}, \sigma_{n}^{2}, l_{n}^{2}, \sigma_{w}^{2}\right\}$ defining $k(\cdot, \cdot)$ and $k_{n}(\cdot, \cdot)$ need to be estimated. This can be done by maximizing the evidence (or log marginal likelihood) given by

$$
\log p\left(\mathbf{x} \mid\left\{T_{i}\right\}_{i}, \boldsymbol{\theta}\right)=-\frac{1}{2} \mathbf{x}^{T}\left(K_{s}+K_{n}\right)^{-1} \mathbf{x}-\frac{1}{2} \log \left|K_{s}+K_{n}\right|-\frac{M}{2} \log (2 \pi) .
$$

The optimization of the latter equation is obtained thanks to a gradient ascent method, assuming that the initial parameter values are not so far from its actual values.

Fetal ECG extraction from a single abdominal sensor is then a direct extension of the proposed method by modelling the recorded signal $x(t)$ as

$$
x(t)=s_{m}(t)+s_{f}(t)+n(t),
$$

where $s_{m}(t)$ is the signal related to the mother, $s_{f}(t)$ is related to the fetus and $n(t)$ is the additive noise. All these signals are modelled by zero-mean GPs with covariance functions $k_{m}(\cdot, \cdot)$ and $k_{f}(\cdot, \cdot)$ defined by $(2)$ and $k_{n}(\cdot, \cdot)$ obtained from (4), respectively. In this case, the estimation of $s_{f}(t)$ is given by

$$
\hat{s}_{f}(t)=\mathbf{k}_{f}^{T}\left(K_{m}+K_{f}+K_{n}\right)^{-1} \mathbf{x},
$$

where $\mathbf{k}_{f}=\left[k_{f}\left(t, T_{1}\right), \cdots, k_{f}\left(t, T_{M}\right)\right]^{T}$.

\section{Numerical experiments}

In this section we first investigate the performance of the proposed method on synthetic data to denoise ECG (Section 4.1). An illustration of f-ECG extraction is then provided on real data (Section 4.2).

\subsection{Synthetic data: ECG denoising}

The performance of the proposed algorithm to denoise ECG is assessed. In the first experiment, each beat of the ECG signal is generated by model (1). To mimic the variability presented in a real ECG, the waves amplitudes and P-R and R$\mathrm{T}$ intervals are randomly changed $(3 \%)$ around their average values. The ECG signal is then obtained as the summation of several beats with random global amplitudes and random R-R intervals. To ensure the consistency of the results, the whole procedure has been repeated one thousand times by regenerating all random parameters of the signal and noise samples. In this experiment, 1500 samples are used with 15 heart beats simulated at $100 \mathrm{~Hz}$ sampled frequency. It is worth noting that the proposed method does not assume that the maxima of the $\mathrm{R}$ peaks are located at observed samples but can also appear in between 


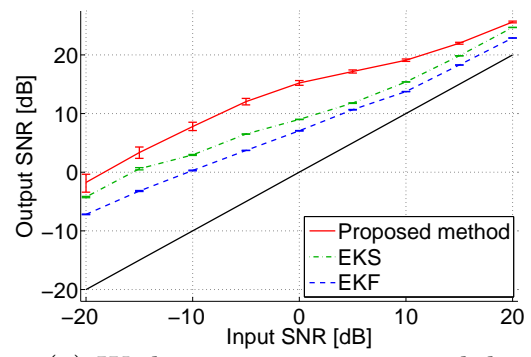

(a) Without parameters variability

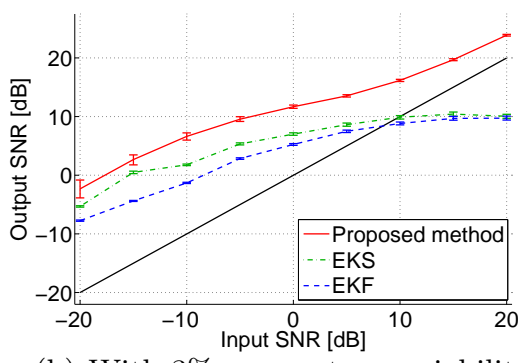

(b) With $3 \%$ parameters variability

Fig. 3. ECG denoising: output SNRs vs. the input SNR without Fig. 3(a) and with Fig. 3(b) parameters variability. In the two figures, the black line corresponds to the same input and output SNRs. In each case, the median are plotted, as well as the first and last quartiles as error bars.

samples. The proposed method is compared to the extended Kalman filtering (EKF) and smoothing (EKS) [13]. The state model is chosen equal to (1) (i.e. the same model than the one used to generate data) whose parameters are equal to average values.

Quantitative results are shown in Fig. 3 which compares the output signal-tonoise ratio (SNR) achieved after denoising versus different input SNRs. As one can see (Fig. 3(b)), the proposed method increases the SNR with a gain between $3 \mathrm{~dB}$ to $18 \mathrm{~dB}$. Contrary to extended Kalman filtering, the proposed method always improves the SNR. Indeed, in the case of high input SNR, EKS and EKF deteriorate the SNR: this can be explained by the variability of the simulated ECG as confirmed by Fig. 3(a), since this phenomenon is not observed without variability. Moreover, one can see that the variability decreases the overall performance, but the proposed method keeps the best performance by a smaller decrease than EKS or EKF.

\subsection{Real data: f-ECG extraction}

In this section, we illustrate (Fig. 4) the proposed method to extract f-ECG from a single sensor on the well-known DaISy fetal ECG database [3]. As one can see, the proposed method provides suitable estimations of both maternal and fetal ECG even when mother's and fetus's R peaks are concomitant (e.g., the fourth, seventh and tenth mother's beats). Moreover, a visual inspection of the residual noise $\hat{n}(t)=x(t)-\hat{s}_{m}(t)-\hat{s}_{f}(t)$ confirms the validity of the assumed modelling (9). Indeed, this residual noise is effectively composed of a smooth varying baseline (dark curve) related to the first term of covariance function (4) plus a quasi white noise (validated by its covariance function empirical estimation). Moreover, both contributions are decorrelated with the mother's and fetus's ECG signals. 


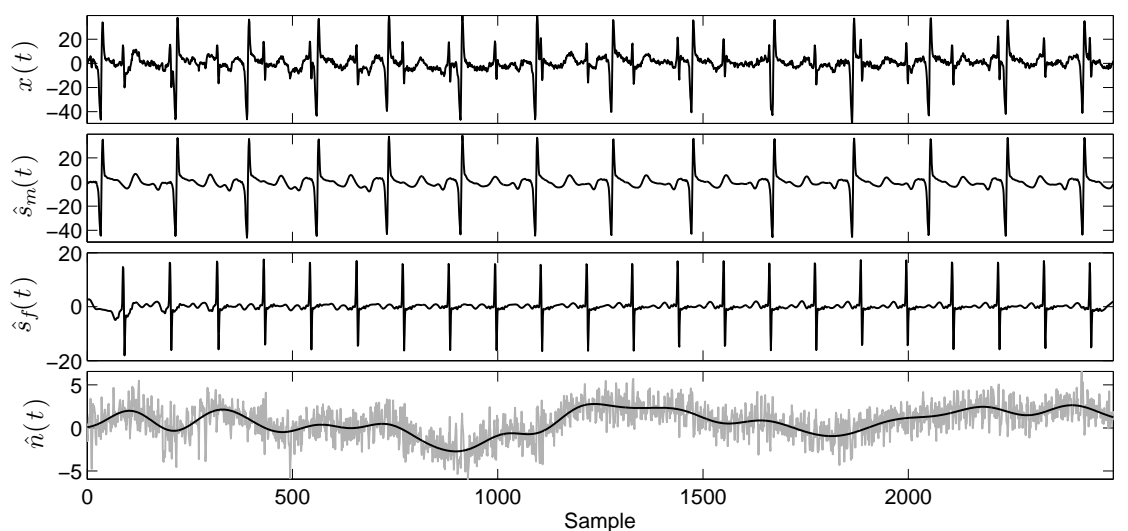

Fig. 4. Fetal ECG extraction. Top to bottom: recorded signal $x(t)$, estimated mother's ECG $\hat{s}_{m}(t)$, estimated fetal's ECG $\hat{s}_{f}(t)$ and residual noise $\hat{n}(t)$ (light gray curve) with estimated baseline (dark curve), respectively.

\section{Conclusions and perspectives}

In this paper, a non-parametric model of ECG signals is derived. By considering them as second order processes, which are fully defined by their mean and covariance functions, one can model a large class of signals with few hyper-parameters. From this modelling, denoising or extraction methods are directly obtained as the maximization of the posterior distribution. Numerical experiments show that the proposed method outperforms an extended Kalman filtering especially in presence of slightly random state parameters. Indeed, Gaussian processes realize a tradeoff between the suitable description of the signal by its second order statistics and its intrinsic variabilities. Finally, the main advantage of the proposed method is its flexibility and it provides a mix between purely data based methods as principal component analysis and parametric model based methods as Kalman filtering.

Future work will deal with a computationally efficient implementation of hyper-parameters estimation of the proposed method as well as an online implementation.

\section{References}

1. M. Cremer. Über die direkte Ableitung der Aktionsströme des menschlichen Herzens vom Ösophagus und über das Elektrokardiogramm des fötus. München. med. Wchnschr., 53:811, 1906.

2. L. De Lathauwer, D. Callaerts, B. De Moor, and J. Vandewalle. Fetal electrocardiogram extraction by source subspace separation. In Proc. IEEE Workshop on HOS, pages 134-138, Girona, Spain, June 12-14 1995. 
3. B. De Moor, P. De Gersem, B. De Schutter, and W. Favoreel. Daisy: A database for identification of systems. Journal A, 38(3):4-5, September 1997.

4. P.P. Kanjilal, S. Palit, and G. Saha. Fetal ecg extraction from single-channel maternal ecg using singular value decomposition. IEEE Transactions on Biomedical Engineering, 44(1):51-59, January 1997.

5. G. S. Kimeldorf and G. Wahba. A correspondence between bayesian estimation on stochastic processes and smoothing by splines. Annal of Mathematical Statistics, 41(2):495-502, 1970.

6. D. J. C. MacKay. Introduction to Gaussian processes. Neural Networks and Machine Learning, 168:1-32, 1998.

7. F. Mochimaru, Y. Fujimoto, and Y. Ishikawa. Detecting the fetal electrocardiogram by wavelet theory-based methods. Progress in Biomedical Research, 7(3):185-193, September 2002.

8. A. Papoulis. Probability, Random Variables, and Stochastic Processes. McGrawHill, third edition, 1991.

9. C. E. Rasmussen and C. K. I. Williams. Gaussian Processes for Machine Learning. MIT Press, 2006.

10. M. Richter, T. Schreiber, and D.T. Kaplan. Fetal ecg extraction with nonlinear state-space projections. IEEE Transactions on Biomedical Engineering, 45(1):133137, January 1998.

11. R. Sameni. Extraction of Fetal Cardiac Signals from an Array of Maternal Abdominal Recordings. PhD thesis, Institut Polytechnique de Grenoble (Grenoble-INP), 2008.

12. R. Sameni and G. D. Clifford. A review of fetal ECG signal processing; issues and promising directions. The Open Pacing, Electrophysiology $\& 3$ Therapy Journal (TOPETJ), 3:4-20, November 2010.

13. R. Sameni, M.B. Shamsollahi, C. Jutten, and G.D. Clifford. A nonlinear bayesian filtering framework for ECG denoising. IEEE Transactions on Biomedical Engineering, 54(12):2172-2185, December 2007.

14. T. Tsalaile, R. Sameni, S. Sanei, C. Jutten, and J. Chambers. Sequential blind source extraction for quasi-periodic signals with time-varying period. IEEE Transactions on Biomedical Engineering, 56(3):646-655, March 2009.

15. B. Widrow, Jr. Glover, J.R., J.M. McCool, J. Kaunitz, C.S. Williams, R.H. Hearn, J.R. Zeidler, Jr. Eugene Dong, and R.C. Goodlin. Adaptive noise cancelling: Principles and applications. Proceedings of the IEEE, 63(12):1692-1716, December 1975.

16. V. Zarzoso and A. K. Nandi. Noninvasive fetal electrocardiogram extraction : blind source separation versus adaptative noise cancellation. IEEE Transactions on Biomedical Engineering, 48(1):12-18, January 2001. 